\title{
Field-free synthetic-ferromagnet spin torque oscillator
}

\author{
Yan Zhou, ${ }^{1}$ Jiang Xiao (萧江), ${ }^{2,3,{ }^{*}}$ Gerrit E. W. Bauer, ${ }^{4,5}$ and F. C. Zhang ${ }^{1,6}$ \\ ${ }^{1}$ Department of Physics, The University of Hong Kong, Hong Kong, China \\ ${ }^{2}$ Department of Physics and State Key Laboratory of Surface Physics, Fudan University, Shanghai, China \\ ${ }^{3}$ Center for Spintronic Devices and Applications, Fudan University, Shanghai, China \\ ${ }^{4}$ Institute for Materials Research and WPI-AIMR, Tohoku University, Sendai, Japan \\ ${ }^{5}$ Kavli Institute of NanoScience, Delft University of Technology, Delft, The Netherlands \\ ${ }^{6}$ Center of Theoretical and Computational Physics, University of Hong Kong, Hong Kong, China \\ (Received 18 October 2012; revised manuscript received 8 December 2012; published 29 January 2013)
}

\begin{abstract}
We study the magnetization dynamics of spin valve structures with a free composite synthetic ferromagnet (SyF) that consists of two ferromagnetic layers coupled through a normal metal spacer. A ferromagnetically coupled SyF can be excited into dynamical precessional states by an applied current without external magnetic fields. We analytically determine the stability of these states in the space spanned by the current density and SyF interlayer exchange coupling. Numerical simulations confirm our analytical results.
\end{abstract}

DOI: 10.1103/PhysRevB.87.020409

PACS number(s): 75.78.-n

The transfer of angular momentum between the magnetic layers of current-driven spin valves (spin-transfer torque) has not been so long ago predicted ${ }^{1,2}$ and experimentally confirmed. ${ }^{3,4}$ The implied efficient electrical control of magnetizations motivated the pursuit of new research directions. When the current density exceeds a critical value, the spintransfer torque can switch the magnetization to a different static configuration without the necessity of applied magnetic fields, which makes it attractive for next generation magnetoresistive random access memory (MRAM) application. ${ }^{3,5-7}$ Under an external magnetic field, the spin-transfer torque can also drive the magnetization into sustainable coherent oscillations spanning a wide frequency range from a few $\mathrm{MHz}$ to several hundred $\mathrm{GHz} .^{3,5-11}$ High frequency magnetic oscillations generate a coherent microwave voltage signal through the giant magnetoresistance (GMR) in metallic spin valves or through the tunneling magnetoresistance (TMR) in magnetic tunnel junctions (MTJs). This effect can be used in so-called spin-torque oscillators (STO), which has many advantages including wide tunability, ${ }^{12}$ very high modulation rates, ${ }^{13,14}$ compact device size, and high compatibility with standard CMOS processes. ${ }^{15,16}$ Thus STO is appealing for high frequency microwave applications including microwave emitters, modulators, and detectors. ${ }^{17}$ However, the necessity of an applied magnetic field up to $\sim 1 \mathrm{~T}$ has severely limited the potential of these STOs for microwave generation and wireless communication applications. Recently, various solutions have been proposed to enable zero-field operation, viz., STO with a perpendicularly magnetized fixed ${ }^{18}$ or spin valves with out-of-plane magnetized free layer, ${ }^{19,20}$ magnetic vortex oscillators, ${ }^{21-27}$ wavy-torque STO by judicially choosing free and fixed layer materials with different spin diffusion lengths, ${ }^{28}$ and a tilted magnetization of the fixed layer with respect to the film plane. ${ }^{29-33}$

Recently, synthetic ferromagnets (SyFs) composed of two ferromagnetic layers separated by a very thin nonmagnetic spacer have been used to replace the free layer of a spin valve or MTJ. ${ }^{34-40}$ SyF based spintronic devices have the advantage of higher thermal stability, smaller stray magnetic fields, faster switching speed, and reduced threshold switching current as compared to single ferromagnetic free layers. ${ }^{34-40}$ Klein et al. $^{40}$ predicted that an antiferromagnetically coupled SyF layer with uncompensated magnetization can generate microwave oscillations at zero applied magnetic field.

Here we predict that a ferromagnetically coupled SyF can also be driven into dynamical precessional states, which, however, are surrounded in parameter space by static canted states with noncollinear magnetizations. We use an analytical approach to determine the stability regimes of the SyF system and confirm results by numerical simulations.

We study a spin torque nanodevice with synthetic ferromagnetic free layers as shown in Fig. 1. The left ferromagnetic film forms the fixed polarizer with magnetization $\mathbf{m}_{0} \| \hat{\mathbf{z}}$, and the SyF consists of two ferromagnetic layers $\mathrm{FM}_{1}$ and $\mathrm{FM}_{2}$ of thickness $d_{1,2}$ with a paramagnetic spacer. The unit vectors describing the magnetization orientation are $\mathbf{m}_{1}$ for $\mathrm{FM}_{1}$ and $\mathbf{m}_{2}$ for $\mathrm{FM}_{2}$. For simplicity we assume that the SyF layers are made of the same materials with identical saturation magnetization $M_{s}$. The exchange coupling strength reads $E_{C}=-J S \mathbf{m}_{1} \cdot \mathbf{m}_{2}$, where $J$ and $S$ are the coupling energy per unit area and the cross section area of the sample, respectively. This corresponds to an effective coupling field $\mathbf{H}_{i}^{\mathbf{c}}=J m_{\bar{i}} /\left(\mu_{0} M_{s} d_{i}\right)$, where $i=1,2$ and $\bar{i}=3-i$, and $\mu_{0}$ is the vacuum magnetic susceptibility. $\mathbf{m}_{1}$ and $\mathbf{m}_{2}$ can be parallel or antiparallel at zero applied field, corresponding to the nonlocal Ruderman-Kittel-Kasuya-Yoshida (RKKY) exchange ferromagnetic $(J>0)$ or antiferromagnetic $(J<0)$ coupling, respectively. The spacer between $\mathrm{FM}_{0}$ and $\mathrm{FM}_{1}$ is presumed thick enough that the RKKY coupling with the fixed layer is negligibly small. Although the dynamic dipolar coupling may be responsible for the apparent reduction of static magnetization ${ }^{41}$ or linewidth of the current-induced spin wave mode, ${ }^{42}$ it is estimated to be much smaller for our case compared to the shape anisotropy field and the other fields due to current-induced spin torque and interlayer exchange coupling and therefore disregarded. ${ }^{40}$

Let $P_{0,1}$ be the spin current polarization by $\mathbf{m}_{0,1}$ such that the spin current density in the two spacers are $P_{0} j$ and $P_{1} j$ with $j$ the electric current density. The corresponding spin-transfer 


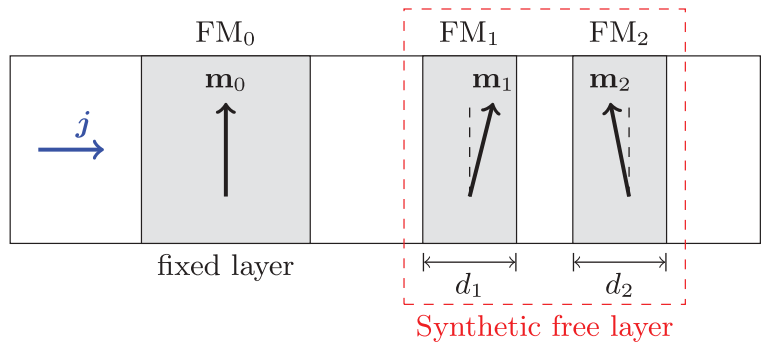

FIG. 1. (Color online) A spin valve structure with an SyF free layer, where $\mathrm{FM}_{0}$ is the fixed layer and $\mathrm{FM}_{1,2}$ layers are (anti) ferromagnetically coupled.

torques on $\mathbf{m}_{1}$ and $\mathbf{m}_{2}$ are given by the projections:

$$
\begin{aligned}
& \mathbf{N}_{\mathrm{ST} 1}=\frac{\gamma \hbar j}{2 e \mu_{0} M_{s} d_{1}} \mathbf{m}_{1} \times\left(P_{0} \mathbf{m}_{0}-P_{1} \mathbf{m}_{2}\right) \times \mathbf{m}_{1}, \\
& \mathbf{N}_{\mathrm{ST} 2}=\frac{\gamma \hbar j}{2 e \mu_{0} M_{s} d_{2}} P_{1} \mathbf{m}_{2} \times \mathbf{m}_{1} \times \mathbf{m}_{2},
\end{aligned}
$$

with $\gamma$ as the gyromagnetic ratio and $P_{0}\left(P_{1}\right)$ are in general functions of the angle $\theta=\angle\left(\mathbf{m}_{0}, \mathbf{m}_{1}\right)\left[\angle\left(\mathbf{m}_{1}, \mathbf{m}_{2}\right)\right] \cdot{ }^{1,43}$

Spin pumping causes enhanced damping in a ferromagnetic layer by emitting spin current into the adjacent nonmagnetic layers. ${ }^{44}$ This emitted spin pumping current can exert a torque on the second layer. Disregarding the backflow and diffusion in the spacer layer, the torque density acting on $\mathbf{m}_{i}$ due to spin pumping from $\mathbf{m}_{\bar{i}}$ can be written as

$$
\mathbf{N}_{\mathrm{SPi}}=\beta \mathbf{m}_{\bar{i}} \times \dot{\mathbf{m}}_{\bar{i}}-\left[\left(\beta \mathbf{m}_{\bar{i}} \times \dot{\mathbf{m}}_{\bar{i}}\right) \cdot \mathbf{m}_{i}\right] \mathbf{m}_{i},
$$

where $\beta$ is the effective enhanced damping due to spin pumping. It has been shown that Eq. (2) gives rise to a dynamic exchange interaction that can induce synchronization of the magnetization dynamics in two neighboring ferromagnetic layers even for wide spacers. ${ }^{45}$ In the results below we fully include the spin pumping. However, in contrast to multilayers excited by microwaves, ${ }^{45}$ we observe here only small corrections demonstrating the dominance of charge current-induced torques.

The dynamics is described by the coupled Landau-LifshitzGilbert-Slonczewski (LLGS) equations, ${ }^{46,47}$

$$
\dot{\mathbf{m}}_{i}=-\gamma \mathbf{m}_{i} \times \mathbf{H}_{i}+\alpha \mathbf{m}_{i} \times \dot{\mathbf{m}}_{i}-\mathbf{N}_{\mathrm{SPi}}-\mathbf{N}_{\mathrm{STi}},
$$

where $\alpha$ is the sum of the intrinsic Gilbert and the spin pumping induced damping. ${ }^{45}$ The effective magnetic fields $\mathbf{H}_{i}$ consist of shape anisotropy and RKKY exchange coupling and can be written as

$$
\mathbf{H}_{i}=\frac{2 K_{u}}{\mu_{0} M_{s}}\left[\mathbf{m}_{i} \cdot \mathbf{e}_{z}\right] \mathbf{e}_{z}+\frac{J \mathbf{m}_{\bar{i}}}{\mu_{0} M_{s} d_{i}} .
$$

For simplicity we consider $d_{1}=d_{2}=d$ (equal magnetization) for the rest of the paper unless otherwise specified. We linearize Eq. (3) in the vicinity of four collinear equilibrium states, i.e., $\uparrow \uparrow, \uparrow \downarrow, \downarrow \uparrow, \downarrow \downarrow$, and assume $\mathbf{m}_{i}=\lambda_{i} \hat{\mathbf{z}}+\mathbf{u}_{i}$ with $\lambda_{i}= \pm$ and $\mathbf{u}_{i}$ denoting the small transverse magnetization component. After the linearization and the Fourier transform $\mathbf{u}_{i}(t)=\int \tilde{\mathbf{u}}_{i}(\omega) e^{-i \omega t} d \omega / 2 \pi$, Eq. (3) becomes

$$
(\hat{A} \omega+\hat{V})\left(\begin{array}{c}
\tilde{\mathbf{u}}_{1} \\
\tilde{\mathbf{u}}_{2}
\end{array}\right)=0,
$$
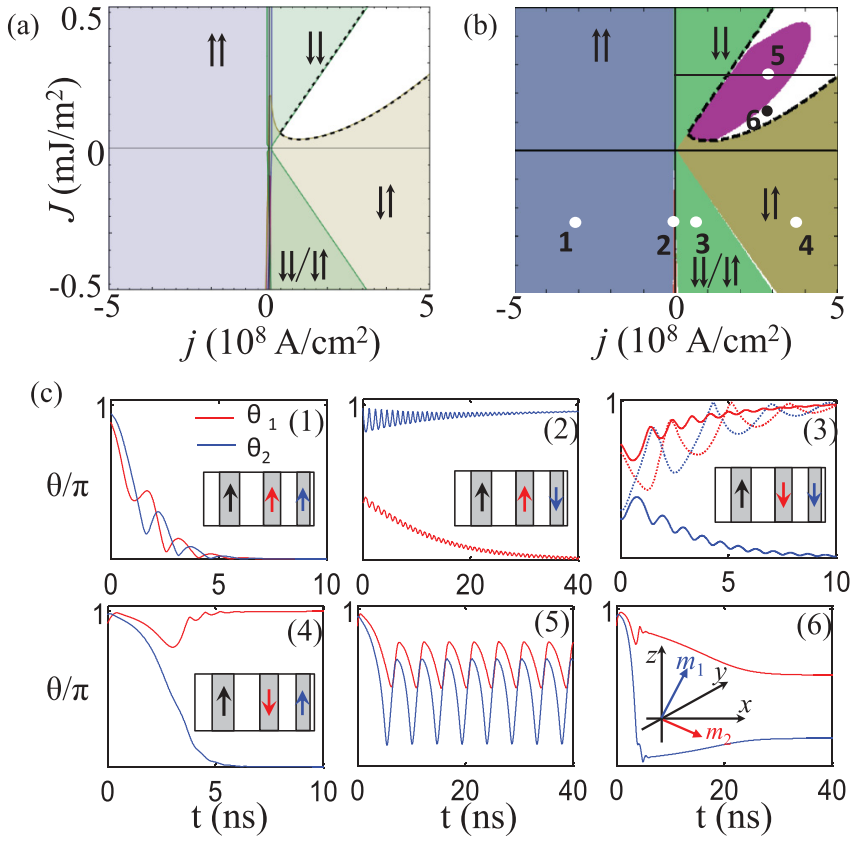

FIG. 2. (Color online) Dynamical phase diagram in the parameter space of currents and RKKY coupling strengths. (a) Phase diagram calculated analytically by Eq. (5); none of the four states $\uparrow \uparrow, \uparrow \downarrow, \downarrow \uparrow, \downarrow \downarrow$ is stable in the white region. (b) Phase diagram calculated by numerically solving the LLGS Eq. (3). The purple are the STO phase, and the white one is the canted state. (c) The time evolution of the polar angles $\theta_{1,2}=\angle\left(\mathbf{m}_{1,2}, \hat{\mathbf{z}}\right)$ at the six different points indicated in the phase diagram. In the third subfigure of (c), the solid and dashed lines correspond to different sets of initial conditions.

with

$$
\begin{aligned}
\hat{A}= & \left(\begin{array}{cc}
1-i \alpha \lambda_{1} & i \beta \lambda_{2} \\
i \beta \lambda_{1} & 1-i \alpha \lambda_{2}
\end{array}\right), \\
\hat{V}= & \omega_{0}\left(\begin{array}{cc}
\lambda_{1} & 0 \\
0 & \lambda_{2}
\end{array}\right)+\omega_{J}\left(\begin{array}{cc}
\lambda_{2} & -\lambda_{1} \\
-\lambda_{2} & \lambda_{1}
\end{array}\right) \\
& +i \omega_{j}\left[P_{0}\left(\begin{array}{cc}
-\lambda_{1} & 0 \\
0 & 0
\end{array}\right)+P_{1}\left(\begin{array}{cc}
\lambda_{1} \lambda_{2} & -1 \\
1 & -\lambda_{1} \lambda_{2}
\end{array}\right)\right],
\end{aligned}
$$

with $\quad \omega_{0}=2 \gamma K_{u} / \mu_{0} M_{s}, \quad \omega_{J}=\gamma J / \mu_{0} M_{s} d, \quad \omega_{j}=$ $(\hbar / 2 e)\left(\gamma j / \mu_{0} M_{s} d\right)$. The frequency of the normal modes are given by the eigenvalues of $\hat{W}=-\hat{A}^{-1} \hat{V}: \Omega_{1}$ and $\Omega_{2}$. When any of the $\operatorname{Im} \Omega_{1,2}>0$, the system is unstable, implying that an infinitesimal perturbation will lead to magnetization dynamics with amplitudes that initially increase exponentially in time.

The above results allow us to calculate the stability regions for the $\uparrow \uparrow, \uparrow \downarrow, \downarrow \uparrow, \downarrow \downarrow$ phases in the space of typical experimental parameters: Angle-independent $P_{1}=P_{2}=P=$ $0.5, d=3 \mathrm{~nm}, K_{u}=8 \times 10^{4} \mathrm{~J} / \mathrm{m}^{3}, j \sim 10^{8} \mathrm{~A} / \mathrm{cm}^{2}$, and $J \sim 1 \mathrm{~mJ} / \mathrm{m}^{2} .{ }^{40,48}$ To analytically construct the stability diagram as shown in the top-left panel of Fig. 2(a), we first calculate the eigenvalues for each given set of $[j, J]$ as given by Eq. (5). Then we determine whether any of the four collinear static states (different combinations of $\left[\lambda_{1}, \lambda_{2}\right]$ ) is stable or not. For example, both the imaginary part of the eigenvalues of $\uparrow \uparrow$ configuration $\left[\lambda_{1}=+1, \lambda_{2}=+1\right]$ are negative when $j \lesssim 0$. Therefore $\uparrow \uparrow$ is stable in the blue region. In this way 

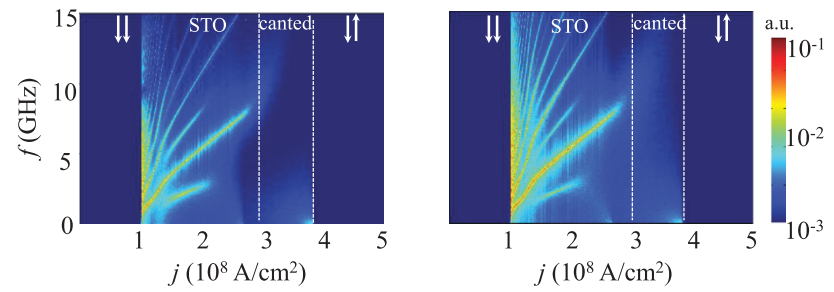

FIG. 3. (Color online) Power spectrum for $\mathbf{m}_{0} \cdot \mathbf{m}_{1}$ (left) and $\mathbf{m}_{1} \cdot \mathbf{m}_{2}$ (right) as a function of current density $j$ and frequency $f$ at $J=0.25 \mathrm{~mJ} / \mathrm{m}^{2}$, corresponding to the black line in the top-right panel of Fig. 2.

we quickly map the parameter space for any given set of $[j, J]$ and construct the entire stability diagram consisting of four collinear magnetization configurations. The spin torque drives the SyF to the parallel $\uparrow \uparrow$ configuration for negative currents $j$. For positive currents, the $\downarrow \uparrow$ configuration is preferred. These results can be understood from Eq. (1). In a small region the antiparallel $\uparrow \downarrow$ state exists for negative $J$ and small $j$ (i.e., in the vicinity of the negative vertical axis but not visible in the figure due to the scale). Although it seems that the $\downarrow \downarrow$ state also occupies the fourth quadrant $(j>0, J<0)$, this triangular region is hysteretic, i.e., $\downarrow \downarrow$ and $\downarrow \uparrow$ may both appear depending on the history.

Most importantly, there is a white/purple region in which none of the four static collinear states is stable, therefore it must be either in a dynamical STO or static canted state. To leading order of $\alpha$ we find from Eq. (5) an approximate boundary for the white region:

$$
\begin{aligned}
& \text { upper: } \omega_{J}=\omega_{j}, \\
& \text { lower: } \omega_{J}=\sqrt{4 \omega_{0}^{2}+\omega_{j}^{2}}-2 \omega_{0}+\alpha \frac{\omega_{0}^{2}}{\omega_{j}},
\end{aligned}
$$

which is plotted as the black dashed lines in Figs. 2(a) and 2(b), matching the numerically obtained boundaries almost exactly. Equation (1) is calculated from the eigenvalue analysis based on Eq. (5) with perturbation from the four static collinear states. This method is equivalent to that used by Bazaliy et al. ${ }^{49}$ A fully analytical solution for the boundary between STO and static canted phase turned out to be intractable due to the complexity of Eq. (5) for noncollinear states.

We now present numerical solutions of the LLGS Eq. (3) including damping, spin torque, and RKKY coupling. We summarize the dynamics of the coupled $\mathbf{m}_{1}$ and $\mathbf{m}_{2}$ in Fig. 2(b), in which we confirm the phase boundaries in the analytical analysis in Fig. 2(a). In addition, we can now map the STO phase by the purple color. The rest of the white region consists of static canted states. In Fig. 2(c) we show the six different SyF configurations that may exist depending on the current and RKKY coupling strength. Point 5 corresponds to an STO state, in which both $\mathbf{m}_{1,2}$ are undergoing large angle precessions, which result in large magnetoresistance oscillations attractive for applications.

For the STO phase we study the power spectrum of the magnetoresistance due to the magnetization oscillation of $\mathbf{m}_{1,2}$, which is approximated by $R(t)=R_{0}+\Delta R_{1} \mathbf{m}_{0} \cdot \mathbf{m}_{1}+$ $\Delta R_{2} \mathbf{m}_{1} \cdot \mathbf{m}_{2}$. Figure 3 shows the Fourier transform of $\mathbf{m}_{0} \cdot \mathbf{m}_{1}$ (left) and $\mathbf{m}_{1} \cdot \mathbf{m}_{2}$ (right) as a function of current density $j$ at $J=0.25 \mathrm{~mJ} / \mathrm{m}^{2}$ [corresponding to the black line in Fig. 2(b)]. The clear higher order harmonic modes are evidence of the nonlinearities in the STO dynamics. Figure 3 also demonstrates that the oscillation frequency of the device can be continuously tuned by the current at zero applied magnetic field and thus potentially be utilized for nanoscale microwave applications. It should be noted that the frequency range can be further tuned by tens of $\mathrm{GHz}$ by adopting a larger $K_{u}$ or taking into account the easy-plane anisotropy field (demagnetization field).

The STO phase studied in this work differs from that studied by Klein et al. ${ }^{40}$ The STO phase found by Klein et al. arises only in an antiferromagnetically $(J<0)$ coupled uncompensated SyF $\left(M_{1}=M_{s} d_{1} S<M_{s} d_{2} S=M_{2}\right)$, in which the total magnetization for the SyF is opposite to that of $\mathbf{m}_{0}$. However, the STO phase found in our study appears in the ferromagnetically coupled SyF with $J>0$ and does not require $M_{1} \neq M_{2}$. Furthermore, we were not able to reproduce the STO phase found by Klein et al. for an uncompensated and antiparallel SyF. We checked the effect of an angular dependence of the prefactor $P_{i}$ that takes into account the effects of a spin accumulation. ${ }^{43}$ The boundaries of the white region will shift noticeably, but we find no qualitative changes. The differences with Ref. 40 might be due to other details in handling spin transport.

Finally, we note that our approach can be readily extended from bilayer to multilayer systems in which each layer is exchange coupled with its neighboring layers (unpublished). This may provide a route to effectively synchronize a large network of spin torque oscillators.

In conclusion, we predict that the ferromagnetically coupled SyF can be driven into STO states without the need of applying magnetic fields. The resulting STO states display large angle precession, therefore generating a large power output. In addition to dynamical STO states, static canted states are also possible in the same structure at slightly different applied current densities. Our findings may guide the experimental effort toward the field-free STO for real applications.

We acknowledge support from University Research Committee (Project No. 106053) of HKU, the University Grant Council (AoE/P-04/08) of the government of HKSAR, the National Natural Science Foundation of China (No. 11004036, No. 91121002), the FOM foundation, DFG Priority Program SpinCat, and EG-STREP MACALO.

\footnotetext{
*Corresponding author: xiaojiang@ fudan.edu.cn

${ }^{1}$ J. C. Slonczewski, J. Magn. Magn. Mater. 159, L1 (1996).

${ }^{2}$ L. Berger, Phys. Rev. B 54, 9353 (1996).

${ }^{3}$ J. Z. Sun, J. Magn. Magn. Mater. 202, 157 (1999).
}

${ }^{4}$ J. A. Katine, F. J. Albert, R. A. Buhrman, E. B. Myers, and D. C. Ralph, Phys. Rev. Lett. 84, 3149 (2000).

${ }^{5}$ T. J. Silva and W. H. Rippard, J. Magn. Magn. Mater. 320, 1260 (2008). 
${ }^{6}$ J. A. Katine and E. E. Fullerton, J. Magn. Magn. Mater. 320, 1217 (2008).

${ }^{7}$ J. Z. Sun and D. C. Ralph, J. Magn. Magn. Mater. 320, 1227 (2008).

${ }^{8}$ M. A. Hoefer, M. J. Ablowitz, B. Ilan, M. R. Pufall, and T. J. Silva, Phys. Rev. Lett. 95, 267206 (2005).

${ }^{9}$ S. I. Kiselev, J. C. Sankey, I. N. Krivorotov, N. C. Emley, R. J. Schoelkopf, R. A. Buhrman, and D. C. Ralph, Nature (London) 425, 380 (2003).

${ }^{10}$ M. Tsoi, A. G. M. Jansen, J. Bass, W. C. Chiang, M. Seck, V. Tsoi, and P. Wyder, Phys. Rev. Lett. 80, 4281 (1998).

${ }^{11}$ M. Tsoi, A. G. M. Jansen, J. Bass, W. C. Chiang, V. Tsoi, and P. Wyder, Nature (London) 406, 46 (2000).

${ }^{12}$ S. Bonetti, P. Muduli, F. Mancoff, and J. Akerman, Appl. Phys. Lett. 94, 102507 (2009).

${ }^{13}$ M. R. Pufall, W. H. Rippard, S. Kaka, T. J. Silva, and S. E. Russek, Appl. Phys. Lett. 86, 082506 (2005).

${ }^{14}$ P. K. Muduli, Y. Pogoryelov, F. Mancoff, and J. Akerman, IEEE Tran. Magn. 47, 1575 (2011).

${ }^{15}$ J. Akerman, Science 308, 508 (2005).

${ }^{16}$ B. N. Engel, J. Akerman, B. Butcher, R. W. Dave, M. DeHerrera, M. Durlam, G. Grynkewich, J. Janesky, S. V. Pietambaram, N. D. Rizzo, J. M. Slaughter, K. Smith, J. J. Sun, and S. Tehrani, IEEE Trans. Magn. 41, 132 (2005).

${ }^{17}$ P. K. Muduli, Y. Pogoryelov, Y. Zhou, F. Mancoff, and J. Akerman, Integrated Ferroelectrics 125, 147 (2011).

${ }^{18}$ D. Houssameddine, U. Ebels, B. Delaet, B. Rodmacq, I. Firastrau, F. Ponthenier, M. Brunet, C. Thirion, J.-P. Michel, L. PrejbeanuBuda, M. C. Cyrille, O. Redon, and B. Dieny, Nat. Mater. 6, 447 (2007).

${ }^{19}$ W. H. Rippard, A. M. Deac, M. R. Pufall, J. M. Shaw, M. W. Keller, S. E. Russek, G. E. W. Bauer, and C. Serpico, Phys. Rev. B 81, 014426 (2010).

${ }^{20}$ S. M. Mohseni, S. R. Sani, J. Persson, T. N. A. Nguyen, S. Chung, Y. Pogoryelov, and J. Akerman, Phys. Stat. Sol.-RRL 5, 432 (2011).

${ }^{21}$ V. S. Pribiag, I. N. Krivorotov, G. D. Fuchs, P. M. Braganca, O. Ozatay, J. C. Sankey, D. C. Ralph, and R. A. Buhrman, Nat. Phys. 3, 498 (2007).

${ }^{22}$ V. S. Pribiag, G. Finocchio, B. J. Williams, D. C. Ralph, and R. A. Buhrman, Phys. Rev. B 80, 180411 (2009).

${ }^{23}$ G. Finocchio, V. S. Pribiag, L. Torres, R. A. Buhrman, and B. Azzerboni, Appl. Phys. Lett. 96, 102508 (2010).

${ }^{24}$ N. Locatelli, V. V. Naletov, J. Grollier, G. de Loubens, V. Cros, C. Deranlot, C. Ulysse, G. Faini, O. Klein, and A. Fert, Appl. Phys. Lett. 98, 062501 (2011).

${ }^{25}$ A. Dussaux, B. Georges, J. Grollier, V. Cros, A. V. Khvalkovskiy, A. Fukushima, M. Konoto, H. Kubota, K. Yakushiji, S. Yuasa, K. A. Zvezdin, K. Ando, and A. Fert, Nat. Comm. 1, 8 (2010).
${ }^{26}$ A. Dussaux, A. V. Khvalkovskiy, J. Grollier, V. Cros, A. Fukushima, M. Konoto, H. Kubota, K. Yakushiji, S. Yuasa, K. Ando, and A. Fert, Appl. Phys. Lett. 98, 132506 (2011).

${ }^{27}$ T. Devolder, Joo-Von Kim, P. Crozat, C. Chappert, M. Manfrini, M. van Kampen, W. Van Roy, L. Lagae, G. Hrkac, and T. Schrefl, Appl. Phys. Lett. 95, 012507 (2009).

${ }^{28}$ O. Boulle, V. Cros, J. Grollier, L. G. Pereira, C. Deranlot, F. Petroff, G. Faini, J. Barnas, and A. Fert, Nat. Phys. 3, 492 (2007).

${ }^{29}$ Y. Zhou, C. L. Zha, S. Bonetti, J. Persson, and J. Akerman, J. Appl. Phys. 105, 07D116 (2009).

${ }^{30}$ Y. Zhou, J. Persson, S. Bonetti, and J. Akerman, Appl. Phys. Lett. 92, 092505 (2008).

${ }^{31}$ Y. Zhou, S. Bonetti, C. L. Zha, and J. Akerman, N. J. Phys. 11, 103028 (2009).

${ }^{32}$ R.-X. Wang, P.-B. He, Z.-D. Li, A.-L. Pan, and Q.-H. Liu, J. Appl. Phys. 109, 033905 (2011).

${ }^{33}$ P.-B. He, R.-X. Wang, Z.-D. Li, W.-M. Liu, A.-L. Pan, Y.-G. Wang, and B.-S. Zou, Eur. Phys. J. B 73, 417 (2010).

${ }^{34}$ T. Taniguchi and H. Imamura, Appl. Phys. Express 4, 103001 (2011).

${ }^{35}$ S. Yakata, H. Kubota, T. Sugano, T. Seki, K. Yakushiji, A. Fukushima, S. Yuasa, and K. Ando, Appl. Phys. Lett. 95, 242504 (2009).

${ }^{36}$ I. Yulaev, M. V. Lubarda, S. Mangin, V. Lomakin, and E. E. Fullerton, Appl. Phys. Lett. 99, 132502 (2011).

${ }^{37} \mathrm{M}$. Ichimura, T. Hamada, H. Imamura, S. Takahashi, and S. Maekawa, J. Appl. Phys. 109, 07C906 (2011).

${ }^{38}$ P. Balaz and J. Barnas, Phys. Rev. B 83, 104422 (2011).

${ }^{39}$ A. Bergman, B. Skubic, J. Hellsvik, L. Nordstrom, A. Delin, and O. Eriksson, Phys. Rev. B 83, 224429 (2011).

${ }^{40}$ C. Klein, C. Petitjean, and X. Waintal, Phys. Rev. Lett. 108, 086601 (2012).

${ }^{41}$ O. Dmytriiev, T. Meitzler, E. Bankowski, A. Slavin, and V. Tiberkevich, J. Phys.: Condens. Matter 22, 136001 (2010).

${ }^{42}$ D. Gusakova, M. Quinsat, J. F. Sierra, U. Ebels, B. Dieny, L. D. Buda-Prejbeanu, M.. C. Cyrille, V. Tiberkevich, and A. N. Slavin, Appl. Phys. Lett. 99, 052501 (2011).

${ }^{43}$ J. Xiao, A. Zangwill, and M. D. Stiles, Phys. Rev. B 70, 172405 (2004).

${ }^{44}$ Y. Tserkovnyak, A. Brataas, and G. E. W. Bauer, Phys. Rev. Lett. 88, 117601 (2002)

${ }^{45}$ Y. Tserkovnyak, A. Brataas, G. E. W. Bauer, and B. I. Halperin, Rev. Mod. Phys. 77, 1375 (2005).

${ }^{46}$ J. Z. Sun, Phys. Rev. B 62, 570 (2000).

${ }^{47}$ J. Xiao, A. Zangwill, and M. D. Stiles, Phys. Rev. B 72, 014446 (2005).

${ }^{48}$ S. Yakata, H. Kubota, T. Seki, K. Yakushiji, A. Fukushima, S. Yuasa, and K. Ando, IEEE T. Magn. 46, 2232 (2010).

${ }^{49}$ Y. B. Bazaliy, B. A. Jones, and S. C. Zhang, Phys. Rev. B 69 , 094421 (2004). 